MATHEMATICS OF COMPUTATION

Volume 71, Number 237, Pages 147-156

S 0025-5718(01)01314-X

Article electronically published on May 7, 2001

\title{
ON THE STABILITY OF THE $L^{2}$ PROJECTION IN $H^{1}(\Omega)$
}

\author{
JAMES H. BRAMBLE, JOSEPH E. PASCIAK, AND OLAF STEINBACH
}

\begin{abstract}
We prove the stability in $H^{1}(\Omega)$ of the $L^{2}$ projection onto a family of finite element spaces of conforming piecewise linear functions satisfying certain local mesh conditions. We give explicit formulae to check these conditions for a given finite element mesh in any number of spatial dimensions. In particular, stability of the $L^{2}$ projection in $H^{1}(\Omega)$ holds for locally quasiuniform geometrically refined meshes as long as the volume of neighboring elements does not change too drastically.
\end{abstract}

\section{InTRODUCTION}

Let $\left\{\varphi_{k}\right\}_{k=1}^{M}$ denote the nodal basis for a piecewise linear (continuous) finite element approximation space $V_{h}$ based on a conforming triangulation (of simplices) $\left\{\tau_{l}\right\}_{l=1}^{N}$ of a polyhedral domain $\Omega$ in $\mathbf{R}^{n}, n=1,2, \ldots$. We shall always assume that the triangulation is locally (but not globally) quasiuniform. The $L^{2}$ projection $Q_{h}$ of a given function $u$ onto the finite element space $V_{h}$ is defined by

$$
\left\langle Q_{h} u, v^{h}\right\rangle_{L^{2}(\Omega)}=\left\langle u, v^{h}\right\rangle_{L^{2}(\Omega)} \quad \text { for all } v^{h} \in V_{h} .
$$

Here $\langle\cdot, \cdot\rangle_{L^{2}(\Omega)}$ denotes the inner product on $L^{2}(\Omega)$. The $L^{2}$ projection is obviously bounded on $L^{2}(\Omega)$; indeed, $\left\|Q_{h} u\right\|_{L^{2}(\Omega)} \leq\|u\|_{L^{2}(\Omega)}$ holds for all $u \in L^{2}(\Omega)$. In this paper we are concerned with the stability of the $L^{2}$ projection as a map $Q_{h}$ : $H^{1}(\Omega) \rightarrow V_{h} \subset H^{1}(\Omega)$. In particular, we will prove the stability estimate

$$
\left\|Q_{h} u\right\|_{H^{1}(\Omega)} \leq c \cdot\|u\|_{H^{1}(\Omega)} \quad \text { for all } u \in H^{1}(\Omega)
$$

under certain conditions on the finite element trial space $V_{h}$, specifically, on the underlying triangulation.

The stability of $Q_{h}$ in $H^{1}(\Omega)$ is of general interest, in particular, for Galerkin finite or boundary element methods for elliptic and parabolic boundary value problems [4 6, 13. For example, the stability estimate (1.2) is needed to analyze the properties of a Neumann series corresponding to a second kind boundary integral equation, see [9].

Using interpolation arguments we get

$$
\left\|Q_{h} u\right\|_{H^{s}(\Omega)} \leq c \cdot\|u\|_{H^{s}(\Omega)} \quad \text { for all } u \in H^{s}(\Omega), \quad s \in[0,1] .
$$

Received by the editor February 11, 2000 and, in revised form, May 24, 2000.

2000 Mathematics Subject Classification. Primary 65D05, 65N30, 65N50.

Key words and phrases. $L^{2}$ projection, finite elements, stability, adaptivity.

This work was supported by the National Science Foundation under grants numbered DMS9626567 and DMS-9973328 and by the State of Texas under ARP/ATP grant \#010366-168. This work was done while the third author was a Postdoctoral Research Associate at the Institute for Scientific Computation (ISC), Texas A \& M University. The financial support by the ISC is gratefully acknowledged. 
From (1.3) we can conclude the stability estimate (see for example [7])

$$
c \cdot\left\|u^{h}\right\|_{H^{s}(\Omega)} \leq \sup _{v^{h} \in V_{h}} \frac{\left|\left\langle u^{h}, v^{h}\right\rangle_{L^{2}(\Omega)}\right|}{\left\|v^{h}\right\|_{\widetilde{H}^{-s}(\Omega)}} \quad \text { for all } u^{h} \in V_{h},
$$

where $\widetilde{H}^{-s}(\Omega)=\left(H^{s}(\Omega)\right)^{\prime}$ is defined by duality with respect to the $L^{2}$ inner product. The estimate (1.4) is essentially needed in the derivation of hybrid coupled finite element domain decomposition methods [1] and for hybrid boundary element methods 11] as well as in the construction of efficient preconditioners in finite and boundary element methods [12].

For globally quasiuniform triangulations, the estimate (1.2) is a direct consequence of a global inverse inequality. In [6], (1.2) was shown for nonuniform triangulations in one and two dimensions satisfying certain mesh conditions. The analysis is based on decay properties of the $L^{2}(\Omega)$ projection and results in conditions which depend on the global behavior of the mesh.

In this paper we prove the stability estimate (1.2) for arbitrary $n=1,2, \ldots$ provided that local stability conditions are satisfied. This approach is valid for more general trial spaces, in particular, for trial functions of arbitrary polynomial degree, but for simplicity, we only consider the case of piecewise linear basis functions. In this case, we formulate explicit local mesh conditions which imply (1.2). These conditions can be easily checked for a given finite element mesh, allowing the user to redefine the mesh if necessary.

The remainder of this paper is organized as follows. Some preliminary notation is given in Section 2. In Section 3 we recall from [5, 8] the definition as well as some error and stability estimates for a quasi interpolation operator needed in our analysis. Our main result is formulated in Theorem 4.1 The proof is based on a general stability condition and several technical results given in Section 5 . In Section 6 we discuss the stability condition in case of piecewise linear basis functions. Based on the eigenvalue analysis of locally defined weighted Gram matrices, we derive computable criteria for guaranteeing that the stability condition is satisfied.

\section{Notation}

Let

$$
\mathcal{T}_{h}=\left\{\tau_{l}\right\}_{l=1}^{N}, \quad \Delta_{l}:=\int_{\tau_{l}} d x \text { for } l=1, \ldots, N
$$

As usual, we consider a family of meshes depending on $h$, the maximum diameter of any simplex. We assume that the triangulations are locally quasiuniform. This means that the diameter of the simplex divided by the diameter of the largest ball contained in the simplex is bounded independently of $h$ for all simplices in all triangulations. Define

$$
h_{l}:=\Delta_{l}^{1 / n} \quad \text { for } l=1, \ldots, N .
$$

Let $\left\{x_{k}\right\}$ the set of all nodes of the mesh $\mathcal{T}_{h}$, where $x_{k}$ is associated to the basis function $\varphi_{k}\left(\varphi_{k}\left(x_{k}\right)=1\right)$. We define

$$
\omega_{k}:=\operatorname{supp} \varphi_{k}, \quad k=1, \ldots, M .
$$


Let $I(k)$ denote the index set of all elements $\tau_{l}$ satisfying $\tau_{l} \subset \omega_{k}$. Then we define a local mesh size associated to the basis function $\varphi_{k}$ by

$$
\hat{h}_{k}:=\frac{1}{\# I(k)} \sum_{l \in I(k)} h_{l} \quad \text { for } k=1, \ldots, M .
$$

Here and in the rest of the paper, \# denotes cardinality. Since the mesh $\mathcal{T}_{h}$ is assumed to be locally quasiuniform, there exists a positive constant $\gamma \geq 1$ not depending on $h$ such that

$$
\gamma^{-1} \leq \frac{\hat{h}_{k}}{h_{l}} \leq \gamma \quad \text { for all } l \in I(k), \quad k=1, \ldots, M .
$$

We define $J(l)$ to be the set of indices of the vertices in $\tau_{l}$. Note that an inverse inequality holds locally [4, i.e.,

$$
\left\|v^{h}\right\|_{H^{1}\left(\tau_{l}\right)} \leq c \cdot h_{l}^{-1} \cdot\left\|v^{h}\right\|_{L^{2}\left(\tau_{l}\right)} \quad \text { for all } v^{h} \in V_{h}, l=1, \ldots, N .
$$

Here and in the remainder of the paper, we use $c$ with or without subscript to denote a generic positive constant which is independent of $h$.

\section{QUASI INTERPOLATION}

To prove the stability estimate (1.2) we need to use a projection operator $P_{h}$ which is stable in $H^{1}(\Omega)$ and which satisfies local error estimates in $L^{2}\left(\tau_{l}\right)$ valid on all finite elements $\tau_{l}$ for $l=1, \ldots, N$. For this we will use the concept of quasi interpolation operators first introduced by Clement in [5]; see also [8].

We define local trial spaces of piecewise linear continuous functions by

$$
V_{h}^{k}:=\left\{\left.v\right|_{\omega_{k}}: v \in V_{h}\right\} .
$$

Let $Q_{h}^{k}$ denote the $L^{2}$ projection onto $V_{h}^{k}$. We clearly have

$$
\begin{aligned}
\left\|Q_{h}^{k} u\right\|_{L^{2}\left(\omega_{k}\right)} & \leq\|u\|_{L^{2}\left(\omega_{k}\right)}, \\
\left\|\left(I-Q_{h}^{k}\right) u\right\|_{L^{2}\left(\omega_{k}\right)} & \leq c \cdot \hat{h}_{k} \cdot|u|_{H^{1}\left(\omega_{k}\right)} .
\end{aligned}
$$

Moreover, since the mesh is assumed to be locally quasiuniform, we have (see, e.g., 3])

$$
\left\|Q_{h}^{k} u\right\|_{H^{1}\left(\omega_{k}\right)} \leq c \cdot\|u\|_{H^{1}\left(\omega_{k}\right)} \quad \text { for all } u \in H^{1}\left(\omega_{k}\right),
$$

for $k=1, \ldots, M$.

Now we define a quasi interpolation operator by

$$
\left(P_{h} u\right)(x)=\sum_{k=1}^{M}\left(Q_{h}^{k} u\right)\left(x_{k}\right) \cdot \varphi_{k}(x) .
$$

It is easy to check that $P_{h}$ is a projection. Moreover, $P_{h}$ is stable in $H^{1}(\Omega)$ and satisfies some local error estimates as asserted in the following lemma.

Lemma 3.1. Let $u$ be in $H^{1}(\Omega)$. There exists a positive constant $c$ independent of $h$ such that

$$
\left\|\left(I-P_{h}\right) u\right\|_{L^{2}\left(\tau_{l}\right)} \leq c \cdot \sum_{k \in J(l)} \hat{h}_{k} \cdot|u|_{H^{1}\left(\omega_{k}\right)} \quad \text { for } l=1, \ldots, N .
$$

Moreover,

$$
\left\|P_{h} u\right\|_{H^{1}(\Omega)} \leq c \cdot\|u\|_{H^{1}(\Omega)} \quad \text { for all } u \in H^{1}(\Omega)
$$


Proof. The proof follows the general ideas already given in [5]. Let $\tau_{l}$ be an arbitrary but fixed finite element and let $\tilde{k} \in J(l)$ be a fixed index. For $x \in \tau_{l}$ we have the representation

$$
\left(P_{h} u\right)(x)=\left(Q_{h}^{\tilde{k}} u\right)(x)+\sum_{k \in J(l), k \neq \tilde{k}}\left[\left(Q_{h}^{k} u\right)\left(x_{k}\right)-\left(Q_{h}^{\tilde{k}} u\right)\left(x_{k}\right)\right] \varphi_{k}(x) .
$$

Let $s=0,1$. Note that

$$
\left\|\varphi_{k}\right\|_{H^{s}\left(\tau_{l}\right)} \leq c \cdot h_{l}^{n / 2-s}
$$

Then, using (3.2) and (3.3), it follows that

$$
\begin{aligned}
\left\|\left(I-P_{h}\right) u\right\|_{H^{s}\left(\tau_{l}\right) \leq} & c_{1} \cdot \hat{h}_{\tilde{k}}^{1-s} \cdot|u|_{H^{1}\left(\omega_{\tilde{k}}\right)} \\
& +c_{2} \cdot h_{l}^{n / 2-s} \sum_{k \in J(l), k \neq \tilde{k}}\left|\left(Q_{h}^{k} u\right)\left(x_{k}\right)-\left(Q_{h}^{\tilde{k}} u\right)\left(x_{k}\right)\right|
\end{aligned}
$$

Now

$$
\left\|v^{h}\right\|_{L_{\infty}\left(\tau_{l}\right)} \leq c \cdot h_{l}^{-n / 2} \cdot\left\|v^{h}\right\|_{L^{2}\left(\tau_{l}\right)} \quad \text { for all } v^{h} \in V_{h}, \quad l=1, \ldots, N .
$$

Thus, (3.2) gives, for $x_{k} \in \tau_{l}$,

$$
\begin{aligned}
\mid\left(Q_{h}^{k} u\right)\left(x_{k}\right)-\left(Q_{h}^{\tilde{k}} u\right) & \left(x_{k}\right) \mid \leq\left\|Q_{h}^{k} u-Q_{h}^{\tilde{k}} u\right\|_{L_{\infty}\left(\tau_{l}\right)} \\
& \leq c \cdot h_{l}^{-n / 2} \cdot\left\|Q_{h}^{k} u-Q_{h}^{\tilde{k}} u\right\|_{L^{2}\left(\tau_{l}\right)} \\
& \leq c \cdot h_{l}^{-n / 2+1} \cdot\left\{|u|_{H^{1}\left(\omega_{k}\right)}+|u|_{H^{1}\left(\omega_{\tilde{k}}\right)}\right\} .
\end{aligned}
$$

Hence,

$$
\left\|\left(I-P_{h}\right) u\right\|_{H^{s}\left(\tau_{l}\right)} \leq c \cdot \sum_{k \in J(l)} \hat{h}_{k}^{1-s} \cdot|u|_{H^{1}\left(\omega_{k}\right)}
$$

for $s=0,1$ and $l=1, \ldots, N$. Using this estimate for $s=0$ gives (3.5), while for $s=1$ we get (3.6) by summing over all elements.

\section{Main Results}

In this section, we will formulate and prove the main result of this paper, the stability estimate (1.2) assuming some appropriate mesh conditions. For this, we define local weights

$$
\gamma_{k}:=\sqrt{\sum_{l \in I(k)} h_{l}^{-2} \cdot\left\|\varphi_{k}\right\|_{L^{2}\left(\tau_{l}\right)}^{2}} \text { for } k=1, \ldots, M .
$$

In addition, for each element $\tau_{l}$, we define local matrices $\mathbf{G}_{l}, \mathbf{D}_{l}$ and $\mathbf{H}_{l}$ by

$$
\mathbf{G}_{l}[j, i]=\left\langle\varphi_{i}^{l}, \varphi_{j}^{l}\right\rangle_{L^{2}\left(\tau_{l}\right)}, \quad \mathbf{D}_{l}=\operatorname{diag}\left(\left\|\varphi_{i}^{l}\right\|_{L^{2}\left(\tau_{l}\right)}^{2}\right), \quad \mathbf{H}_{l}=\operatorname{diag}\left(\hat{h}_{i}^{l}\right),
$$

for $i, j=1,2,3$ Here $\varphi_{i}^{l}$ and $\varphi_{j}^{l}$ are the basis functions corresponding to the $i$ th and $j$ th vertex of the $l$ th element, respectively, while $\hat{h}_{i}^{l}$ is the related value of $\hat{h}$.

Now we are able to formulate local conditions to be used in the remainder of this section, specifically,

$$
\left(\mathbf{H}_{l}^{-1} \mathbf{G}_{l} \mathbf{H}_{l} \underline{x}^{l}, \underline{x}^{l}\right) \geq c_{0} \cdot\left(\mathbf{D}_{l} \underline{x}^{l}, \underline{x}^{l}\right) \quad \text { for all } \underline{x}^{l} \in \mathbf{R}^{\# J(l)} .
$$

Here $(\cdot, \cdot)$ denotes the inner product on $\mathbf{R}^{\# J(l)}$. 
Remark 4.1. Let $H$ denote the diagonal matrix,

$$
H=\operatorname{diag}\left(\hat{h}_{k}\right) \text {. }
$$

For $\underline{u} \in \mathbf{R}^{M}$, define $\underline{v}=H \underline{u}$ and $\underline{w}=H^{-1} \underline{u}$. The corresponding finite element functions are given by

$$
u^{h}=\sum_{k=1}^{M} \underline{u}_{k} \varphi_{k}, \quad v^{h}=\sum_{k=1}^{M} \underline{v}_{k} \varphi_{k}, \quad w^{h}=\sum_{k=1}^{M} \underline{w}_{k} \varphi_{k} .
$$

We note that $\left(\mathbf{H}_{l}^{-1} \mathbf{G}_{l} \mathbf{H}_{l} \underline{u}^{l}, \underline{u}^{l}\right)=\left\langle v^{h}, w^{h}\right\rangle_{L^{2}\left(\tau_{l}\right)}$, where $\underline{u}^{l}$ denotes the components of $\underline{u}$ associated with the element $\tau_{l}$. By local quasiuniformity, (4.2) is thus equivalent to

$$
\left\|u^{h}\right\|_{L^{2}\left(\tau_{l}\right)}^{2} \leq c\left\langle v^{h}, w^{h}\right\rangle_{L^{2}\left(\tau_{l}\right)} .
$$

If all of the simplices have the same measure then $\mathbf{H}_{l}^{-1} \mathbf{G}_{l} \mathbf{H}_{l}=\mathbf{G}_{l}$, so

$$
\left\langle v^{h}, w^{h}\right\rangle_{L^{2}\left(\tau_{l}\right)}=\left\langle u^{h}, u^{h}\right\rangle_{L^{2}\left(\tau_{l}\right)}
$$

and (4.3) is trivial. The inequality will still hold provided that the measures of neighboring simplices do not vary too much. Explicit local conditions on the mesh for the case of piecewise linear elements are given in Section 6 .

The following result is the main theorem of this paper.

Theorem 4.1. Let condition (4.2) be satisfied. Then the $L^{2}$ projection $Q_{h}: H^{1}(\Omega)$ $\rightarrow V_{h} \subset H^{1}(\Omega)$ is stable. In particular, there exists a positive constant $c$ independent of $h$ such that

$$
\left\|Q_{h} v\right\|_{H^{1}(\Omega)} \leq c \cdot\|v\|_{H^{1}(\Omega)} \quad \text { for all } v \in H^{1}(\Omega) .
$$

The proof of this theorem depends on the following lemma. A similar estimate was used in [2] to construct spectrally equivalent multilevel preconditioners in finite element methods in the case of globally quasiuniform meshes. The proof of the lemma will be given in the next section.

Lemma 4.1. Let condition (4.2) be satisfied. Then there exists a positive constant c such that

$$
\sum_{l=1}^{N} h_{l}^{-2} \cdot\left\|v^{h}\right\|_{L^{2}\left(\tau_{l}\right)}^{2} \leq c \cdot \sum_{k=1}^{M}\left[\frac{\left\langle v^{h}, \varphi_{k}\right\rangle_{L^{2}(\Omega)}}{\hat{h}_{k} \cdot\left\|\varphi_{k}\right\|_{L^{2}(\Omega)}}\right]^{2}
$$

for all $v^{h} \in V_{h}$.

Proof of Theorem [4.1. Using the triangle inequality, (3.6), and (2.6), we get

$$
\begin{aligned}
\left\|Q_{h} v\right\|_{H^{1}(\Omega)}^{2} & \leq 2 \cdot\left\{\left\|P_{h} v\right\|_{H^{1}(\Omega)}^{2}+\sum_{l=1}^{N}\left\|\left(Q_{h}-P_{h}\right) v\right\|_{H^{1}\left(\tau_{l}\right)}^{2}\right\} \\
& \leq c \cdot\left\{\|v\|_{H^{1}(\Omega)}^{2}+\sum_{l=1}^{N} h_{l}^{-2} \cdot\left\|\left(Q_{h}-P_{h}\right) v\right\|_{L^{2}\left(\tau_{l}\right)}^{2}\right\} .
\end{aligned}
$$


From Lemma 4.1 and the Schwarz inequality it follows that

$$
\begin{aligned}
\sum_{l=1}^{N} h_{l}^{-2} \cdot\left\|\left(Q_{h}-P_{h}\right) v\right\|_{L^{2}\left(\tau_{l}\right)}^{2} & \leq c \cdot \sum_{k=1}^{M}\left[\frac{\left\langle\left(Q_{h}-P_{h}\right) v, \varphi_{k}\right\rangle_{L^{2}(\Omega)}}{\hat{h}_{k} \cdot\left\|\varphi_{k}\right\|_{L^{2}(\Omega)}}\right]^{2} \\
& =c \cdot \sum_{k=1}^{M}\left[\frac{\left\langle\left(I-P_{h}\right) v, \varphi_{k}\right\rangle_{L^{2}\left(\omega_{k}\right)}}{\hat{h}_{k} \cdot\left\|\varphi_{k}\right\|_{L^{2}(\Omega)}}\right]^{2} \\
& \leq c \cdot \sum_{k=1}^{M} \hat{h}_{k}^{-2} \cdot\left\|\left(I-P_{h}\right) v\right\|_{L^{2}\left(\omega_{k}\right)}^{2}
\end{aligned}
$$

Hence, the assertion follows from 3.5.

\section{Proof of Lemma 4.1}

In this section, we prove Lemma 4.1. We start by introducing some notation. We define two additional $M \times M$ diagonal matrices given by

$$
D_{\gamma}=\operatorname{diag}\left(\gamma_{k}\right), \quad D_{\varphi}=\operatorname{diag}\left(\hat{h}_{k} \cdot\left\|\varphi_{k}\right\|_{L^{2}(\Omega)}\right)
$$

where $\hat{h}_{k}$ and $\gamma_{k}$ are defined as in (2.4) and (4.1), respectively. The first step in the proof of Lemma 4.1 involves estimating the inverse of a scaled Gram matrix.

Lemma 5.1. Let assumption (4.2) be satisfied. Then there exists a positive constant $c$ such that

$$
\|\underline{x}\|_{2} \leq c \cdot\|A \underline{x}\|_{2} \quad \text { for all } \underline{x} \in \mathbf{R}^{M},
$$

where $A$ is the scaled Gram matrix defined by

$$
A=D_{\varphi}^{-1} G D_{\gamma}^{-1}
$$

and $G$ is the Gram matrix $G_{i j}=\left\langle\varphi_{i}, \varphi_{j}\right\rangle_{L^{2}(\Omega)}$.

Proof. Let $\underline{u}, \underline{u^{l}}, \underline{v}, \underline{w}, u^{h}, v^{h}$ and $w^{h}$ be as in Remark 4.1. Setting $\widetilde{G}=H^{-1} G H$ and using (4.2) gives

$$
\begin{aligned}
(\widetilde{G} \underline{u}, \underline{u}) & =(G \underline{v}, \underline{w})=\left\langle v^{h}, w^{h}\right\rangle_{L^{2}(\Omega)}=\sum_{l=1}^{N}\left\langle v^{h}, w^{h}\right\rangle_{L^{2}\left(\tau_{l}\right)} \\
& =\sum_{l=1}^{N}\left(\mathbf{H}_{l}^{-1} \mathbf{G}_{l} \mathbf{H}_{l} \underline{u}^{l}, \underline{u}^{l}\right) \geq c_{0} \cdot \sum_{l=1}^{N}\left(\mathbf{D}_{l} \underline{u}^{l}, \underline{u}^{l}\right)=c_{0} \cdot(D \underline{u}, \underline{u}) .
\end{aligned}
$$

Here $D$ is the diagonal matrix with entries $\left\|\varphi_{k}\right\|_{L^{2}(\Omega)}^{2}$. Let $D^{1 / 2}=\operatorname{diag}\left(\left\|\varphi_{k}\right\|_{L^{2}(\Omega)}\right)$. From

$$
\begin{aligned}
c_{0} \cdot\left\|D^{1 / 2} \underline{u}\right\|_{2}^{2} & =c_{0} \cdot(D \underline{u}, \underline{u}) \leq(\widetilde{G} \underline{u}, \underline{u}) \\
& =\left(D^{-1 / 2} \widetilde{G} \underline{u}, D^{1 / 2} \underline{u}\right) \leq\left\|D^{-1 / 2} \widetilde{G} \underline{u}\right\|_{2}\left\|D^{1 / 2} \underline{u}\right\|_{2},
\end{aligned}
$$

we conclude that

$$
c_{0} \cdot\left\|D^{1 / 2} \underline{u}\right\|_{2} \leq\left\|D^{-1 / 2} \widetilde{G} \underline{u}\right\|_{2} \quad \text { for all } \underline{u} \in \mathbf{R}^{M} .
$$

Taking $\underline{\tilde{u}}=D_{\gamma} \underline{u}$ gives

$$
c_{0} \cdot\left\|D^{1 / 2} D_{\gamma}^{-1} \underline{\tilde{u}}\right\|_{2} \leq\left\|D^{-1 / 2} D_{\varphi} D_{\varphi}^{-1} \widetilde{G} D_{\gamma}^{-1} \underline{\tilde{u}}\right\|_{2}=\left\|D^{-1 / 2} D_{\varphi} \widetilde{\widetilde{A}} \underline{\tilde{u}}\right\|_{2},
$$


where $\widetilde{A}=D_{\varphi}^{-1} \widetilde{G} D_{\gamma}^{-1}$. The ratio of the diagonal entries satisfies

$$
\frac{D^{1 / 2}[k, k]}{D_{\gamma}[k, k]}=\frac{\left\|\varphi_{k}\right\|_{L^{2}(\Omega)}}{\sqrt{\sum_{l \in I(k)} h_{l}^{-2}\left\|\varphi_{k}\right\|_{L^{2}\left(\tau_{l}\right)}^{2}}} \geq c \cdot \hat{h}_{k}
$$

and

$$
\frac{D_{\varphi}[k, k]}{D^{1 / 2}[k, k]}=\frac{\hat{h}_{k} \cdot\left\|\varphi_{k}\right\|_{L^{2}(\Omega)}}{\left\|\varphi_{k}\right\|_{L^{2}(\Omega)}}=\hat{h}_{k}
$$

for all $k=1, \ldots, M$. Thus,

$$
c \cdot\|H \underline{\tilde{u}}\|_{2} \leq\|H \tilde{A} \underline{\tilde{u}}\|_{2} \quad \text { for all } \underline{\tilde{u}} \in \mathbf{R}^{M} .
$$

Taking $\underline{x}=H \underline{\tilde{u}}$ above gives

$$
c \cdot\|\underline{x}\|_{2} \leq\left\|H \widetilde{A} H^{-1} \underline{x}\right\|_{2}=\left\|H D_{\varphi}^{-1} H^{-1} G H D_{\gamma}^{-1} H^{-1} \underline{x}\right\|_{2}=\|A \underline{x}\|_{2}
$$

for all $\underline{x} \in \mathbf{R}^{M}$. This completes the proof of the lemma.

We now give the proof of Lemma 4.1

Proof of Lemma 4.1. Let $\underline{v} \in \mathbf{R}^{M}$ and set $v^{h}=\sum_{k=1}^{M} \underline{v}_{k} \varphi_{k} \in V_{h}$. Then, the left hand side of (4.5) is bounded by

$$
\begin{aligned}
\sum_{l=1}^{N} h_{l}^{-2} \cdot\left\|v^{h}\right\|_{L^{2}\left(\tau_{l}\right)}^{2} & \leq c \cdot \sum_{l=1}^{N} h_{l}^{-2} \sum_{k \in J(l)} \underline{v}_{k}^{2} \cdot\left\|\varphi_{k}\right\|_{L^{2}\left(\tau_{l}\right)}^{2} \\
& =c \cdot \sum_{k=1}^{M} \underline{v}_{k}^{2} \sum_{l \in I(k)} h_{l}^{-2} \cdot\left\|\varphi_{k}\right\|_{L^{2}\left(\tau_{l}\right)}^{2} \\
& =c \cdot \sum_{k=1}^{M} \underline{v}_{k}^{2} \gamma_{k}^{2}=c \cdot \sum_{k=1}^{M} \underline{x}_{k}^{2}=c \cdot\|\underline{x}\|_{2}^{2},
\end{aligned}
$$

where $\underline{x}_{k}=\gamma_{k} \underline{v}_{k}$. The right hand side in (4.5) is

$$
\begin{aligned}
\sum_{k=1}^{M}\left[\frac{\left\langle v^{h}, \varphi_{k}\right\rangle_{L^{2}(\Omega)}}{\hat{h}_{k}\left\|\varphi_{k}\right\|_{L^{2}(\Omega)}}\right]^{2} & =\sum_{k=1}^{M}\left[\sum_{j=1}^{M} \underline{v}_{j} \cdot \frac{\left\langle\varphi_{j}, \varphi_{k}\right\rangle_{L^{2}(\Omega)}}{\hat{h}_{k}\left\|\varphi_{k}\right\|_{L^{2}(\Omega)}}\right]^{2} \\
& =\sum_{k=1}^{M}\left[\sum_{j=1}^{M} \underline{x}_{j} \cdot \frac{\left\langle\varphi_{j}, \varphi_{k}\right\rangle_{L^{2}(\Omega)}}{\gamma_{j} \hat{h}_{k}\left\|\varphi_{k}\right\|_{L^{2}(\Omega)}}\right]^{2} \\
& =\sum_{k=1}^{M}\left[(A \underline{x})_{k}\right]^{2}=\|A \underline{x}\|_{2}^{2},
\end{aligned}
$$

using the matrix definition (5.7). Hence, (4.5) follows from Lemma 5.1.

Although we only considered the case of piecewise linear basis functions, the same approach may be used for higher order piecewise polynomial finite element spaces. In addition, the case of $V_{h} \subset H_{0}^{1}(\Omega)$ with basis functions vanishing along the boundary $\partial \Omega$ can be treated with only slight modifications. In this case we consider the index set $I(k)$ only for nodes $x_{k}$ associated with a basis function $\varphi_{k} \in V_{h}$. Then all proofs given above apply. Note that the dimension of the local 
matrices for boundary simplices decrease since the rows and columns corresponding to boundary nodes do not appear.

\section{Finite element SPaCeS}

The stability estimate in Theorem 4.1 is based on the condition (4.2). If we define the symmetric matrix

$$
\mathbf{G}_{l}^{S}:=\frac{1}{2}\left[\mathbf{H}_{l} \mathbf{G}_{l} \mathbf{H}_{l}^{-1}+\mathbf{H}_{l}^{-1} \mathbf{G}_{l} \mathbf{H}_{l}\right]
$$

then (4.2) is the same as

$$
\left(\mathbf{G}_{l}^{S} \underline{x}_{l}, \underline{x}_{l}\right) \geq c_{0} \cdot\left(\mathbf{D}_{l} \underline{x}_{l}, \underline{x}_{l}\right) \quad \text { for all } \underline{x}_{l} \in \mathbf{R}^{\# J(l)} .
$$

Let $\tau_{l}$ in $\mathcal{T}_{h}$ be an arbitrary element. Note that $\# J(l)=n+1$. A simple computation shows that

$$
\mathbf{D}_{l}=d_{n} \cdot \Delta_{l} \cdot I \quad \text { with } d_{n}=\frac{2}{(n+1)(n+2)},
$$

where $I$ is the identity matrix in $n+1$ dimensions. Moreover,

$$
\mathbf{G}_{l}^{S}=\frac{1}{4} \cdot d_{n} \cdot \Delta_{l} \cdot \mathbf{A}_{l},
$$

where the matrix $\mathbf{A}_{l}$ is defined by

$$
\mathbf{A}_{l}[i, j]=\left\{\begin{array}{cl}
4 & \text { for } i=j, \\
\frac{\hat{h}_{i}^{l}}{\hat{h}_{j}^{l}}+\frac{\hat{h}_{j}^{l}}{\hat{h}_{i}^{l}} & \text { for } i \neq j,
\end{array} \quad i, j=1, \ldots, n+1 .\right.
$$

Here $\hat{h}_{j}^{l}$ is the value of $\hat{h}$ corresponding to the $j$ th vertex of the $l$ th element. Hence, to show (6.2) it is sufficient to consider the eigenvalues $\left\{\lambda_{i}\right\}$ of the matrix $\mathbf{A}_{l}$. To this end, we give the following proposition.

Proposition 6.1. Let $\alpha_{1}, \ldots, \alpha_{n+1}$ be real numbers with $\alpha_{i} \neq 0$ for $i=1, \ldots$, $n+1$, and consider the matrix

$$
\mathbf{A}[i, j]=\left\{\begin{array}{cl}
4 & \text { for } i=j, \\
\frac{\alpha_{i}}{\alpha_{j}}+\frac{\alpha_{j}}{\alpha_{i}} & \text { for } i \neq j,
\end{array} \quad i, j=1, \ldots, n+1 .\right.
$$

Then, all eigenvalues of $\mathbf{A}$ are in the set $\left\{\lambda_{+}, \lambda_{-}, 2\right\}$, where

$$
\lambda_{ \pm}=3+n \pm \sqrt{\sum_{i=1}^{n+1} \alpha_{i}^{2} \cdot \sum_{i=1}^{n+1} \alpha_{i}^{-2}}
$$

Proof. We first consider the case when $\alpha_{1}, \alpha_{2}, \ldots, \alpha_{n+1}$ are not all equal. Let $\mathbf{M}_{+}=\left(\alpha_{1}, \alpha_{2}, \ldots, \alpha_{n+1}\right)^{t}$ and $\mathbf{M}_{-}=\left(\alpha_{1}^{-1}, \alpha_{2}^{-1}, \ldots, \alpha_{n+1}^{-1}\right)^{t}$. Then $\mathbf{A}=2 I+\mathbf{N}$, where $\mathbf{N}=\mathbf{M}_{-} \cdot \mathbf{M}_{+}^{t}+\mathbf{M}_{+} \cdot \mathbf{M}_{-}^{t}$. Note that the matrix $\mathbf{N}$ is symmetric with range equal to the two dimensional subspace spanned by $\mathbf{M}_{+}$and $\mathbf{M}_{-}$. Thus, zero is an eigenvalue of $\mathbf{N}$ with multiplicity $n-1$. We need only compute the two remaining eigenvalues. By expanding the corresponding eigenvectors in the basis $\left\{\mathbf{M}_{+}, \mathbf{M}_{-}\right\}$, 
it is elementary to see that the remaining two eigenvalues of $\mathbf{N}$ are eigenvalues of the matrix

$$
\left(\begin{array}{cc}
n+1 & \sum_{i=1}^{n+1} \alpha_{i}^{2} \\
\sum_{i=1}^{n+1} \alpha_{i}^{-2} & n+1
\end{array}\right)
$$

The proposition immediately follows for this case.

If $\alpha_{1}=\alpha_{2}=\cdots=\alpha_{n+1}$ then $\mathbf{M}_{+}$and $\mathbf{M}_{-}$are linearly dependent, so 2 is an eigenvalue of $\mathbf{A}$ with multiplicity $n$. The remaining eigenvalue is $\lambda_{+}=2 n+4$. This completes the proof of the proposition.

It follows from (6.4) that the local condition (6.2) is satisfied if all eigenvalues of $\mathbf{A}_{l}$ are strictly positive and bounded away from zero. By the proposition, this is equivalent to

$$
3+n-\sqrt{\sum_{i=1}^{n+1}\left(\hat{h}_{i}^{l}\right)^{-2} \cdot \sum_{i=1}^{n+1}\left(\hat{h}_{i}^{l}\right)^{2}} \geq c
$$

with $c$ independent of $\tau_{l}$. Inequality (6.6) provides a mesh constraint for adaptive triangulations.

Remark 6.1. We can satisfy (6.6) for any $0<c<2$ provided that we design a mesh which gives rise to a $\gamma$ (in (2.5) ) sufficiently close to one. It immediately follows from (2.5) that

$$
3+n-\sqrt{\sum_{i=1}^{n+1}\left(\hat{h}_{i}^{l}\right)^{-2} \cdot \sum_{i=1}^{n+1}\left(\hat{h}_{i}^{l}\right)^{2}} \geq 2-(n+1)\left(\gamma^{2}-1\right) .
$$

Thus (6.6) holds if

$$
\gamma \leq \sqrt{1+\frac{2-c}{n+1}}
$$

Thus, a mesh gives rise to an $H^{1}(\Omega)$ stable $L^{2}$ projection provided that the change in measures of neighboring simplices are controlled.

Finally, if a finite element mesh $\mathcal{T}_{h}$ is given, the mesh condition (6.6) and therefore the stability assumption (4.2) can be checked explicitly (by direct computation). To illustrate the applicability of the mesh condition (6.6), we consider an adaptive finite element mesh for $n=2$ as shown in Figure 1, generated by an adaptive algorithm described in [10. In Table 1 we give values for

$$
c=\max _{\tau_{l}}\left(5-\sqrt{\sum_{i=1}^{3}\left(\hat{h}_{i}^{l}\right)^{-2} \cdot \sum_{i=1}^{3}\left(\hat{h}_{i}^{l}\right)^{2}}\right)
$$

as a function of the refinement level $L$ and the number of finite element nodes $M$.

TABle 1. Computational results for $c$

\begin{tabular}{|c|c|c|c|c|c|c|c|c|c|c|}
\hline $\mathrm{L}$ & 0 & 1 & 2 & 3 & 4 & 5 & 6 & 7 & 8 & 9 \\
\hline $\mathrm{M}$ & 8 & 17 & 28 & 53 & 87 & 155 & 291 & 532 & 1034 & 2003 \\
\hline$c$ & 2.00 & 1.93 & 1.59 & 1.52 & 1.52 & 1.66 & 1.61 & 1.09 & 1.49 & 1.50 \\
\hline
\end{tabular}




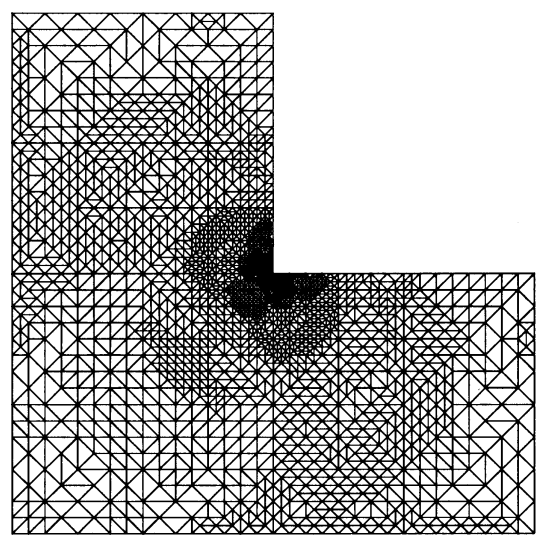

Figure 1. Adaptive finite element triangulation

\section{REFERENCES}

[1] A. Agouzal, J.-M. Thomas, Une methode d'elements finis hybrides en decomposition de domains. Math. Modell. Numer. Anal. 29 (1995) 749-764. MR 96g:65115

[2] J. H. Bramble, J. E. Pasciak, P. S. Vassilevski, Computational scales of Sobolev norms with applications to preconditioning. Math. Comp. 69 (2000), 463-480. MR 2000k:65088

[3] J. H. Bramble, J. Xu, Some estimates for a weighted $L^{2}$ projection. Math. Comp. 56 (1991) 463-476. MR 91i:65140

[4] P. G. Ciarlet, The Finite Element Method for Elliptic Problems. North-Holland, 1978. MR 58:25001

[5] P. Clement, Approximation by finite element functions using local regularization. RAIRO Anal. Numer. 9 R-2 (1975) 77-84. MR 53:4569

[6] M. Crouzeix, V. Thomeé, The stability in $L_{p}$ and $W_{p}^{1}$ of the $L^{2}$-projection onto finite element function spaces. Math. Comp. 48 (1987) 521-532. MR 88f:41016

[7] W. McLean, O. Steinbach, Boundary element preconditioners for a hypersingular integral equation on a curve. Adv. Comput. Math. 11 (1999) 271-286. MR 2000k:65236

[8] L. R. Scott, S. Zhang, Finite element interpolation of nonsmooth functions satisfying boundary conditions. Math. Comp. 54 (1990) 483-493. MR 90j:65021

[9] H. Schulz, O. Steinbach, A new a posteriori error estimator in direct boundary element methods. The Neumann problem. Multifield Problems. State of the Art. (A.-M. Sändig, W. Schiehlen, and W. L. Wendland, eds.) Springer-Verlag, Berlin, 201-208, 2000.

[10] O. Steinbach, Adaptive finite element-boundary element solution of boundary value problems. J. Comput. Appl. Math. 106 (1999) 307-316. MR 2000b:65225

[11] O. Steinbach, On a hybrid boundary element method. Numer. Math. 84 (2000), 679-695. MR 2001a:65154

[12] O. Steinbach, W. L. Wendland, The construction of some efficient preconditioners in the boundary element method. Adv. Comput. Math. 9 (1998) 191-216. MR 99j:65219

[13] L. B. Wahlbin, Superconvergence in Galerkin Finite Element Methods. Lecture Notes in Mathematics 1605, Springer, Berlin, 1995. MR 98j:65083

Department of Mathematics, Texas A \& M University, College Station, Texas 77843

E-mail address: bramble@math.tamu.edu

Department of Mathematics, Texas A \& M University, College Station, Texas 77843

E-mail address: pasciak@math.tamu.edu

Mathematisches Institut A, Universität Stuttgart, Pfaffenwaldring 57, 70569 Stuttgart, Germany

E-mail address: steinbach@mathematik.uni-stuttgart.de 